\title{
Corrigendum
}

\section{Prenatal intimate partner violence exposure predicts infant biobehavioral regulation: Moderation by the brain-derived neurotrophic factor (BDNF) gene - CORRIGENDUM}

Cecilia Martinez-Torteya, Caleb J. Figge, Michelle A. Gilchrist, Maria Muzik, Anthony P. King and Matthew Sorenson

doi: $10.1017 /$ S0954579418000329, published by Cambridge University Press, 2 August 2018

The original publication of this article (Martinez-Torteya et al., 2018) contained a typographical error in Martinez-Torteya's affiliation. The article has since been corrected.

\section{Reference}

Martinez-Torteya, C., Figge, C.J., Gilchrist, M.A., Muzik, M., King, A.P., \& Sorenson, M. Prenatal intimate partner violence exposure predicts infant biobehavioral regulation: Moderation by the brain-derived neurotrophic factor (BDNF) gene. Development and Psychopathology, 30(3), 1009-1021. doi: $10.1017 /$ S0954579418000329 